\title{
A dynamic update of the HWMP proactive tree for IEEE 802.11s based wireless mesh networks
}

\author{
Mohamed GUESMIA, Mustapha GUEZOURI, and Nader MBAREK
}

\begin{abstract}
In this paper we propose a dynamic update of the HWMP (Hybrid Wireless Mesh Protocol) proactive tree by changing dynamically the path request (PREQ) transmission interval, instead of a periodic fixed value as defined in the IEEE 802.11s draft. Indeed, we adapt dynamically the value of the PREQ transmission interval according to the wireless environment characteristics and to the amount of traffic flows generated by applications within the considered environment. Thereby, we expect improving the routing table accuracy and minimizing the path recovery delays with less overhead for critical applications. Simulations results using Network Simulator 3 (NS-3) show that our proposed dynamic and non periodic PREQ transmission interval gives better results than a fixed periodic transmission interval value.
\end{abstract}

Keywords-Wireless Mesh Networks, IEEE 802.11s, HWMP proactive tree, Unicast and Multicast communications.

\section{INTRODUCTION}

The Wireless Mesh Network (WMN) is an emerging technology deployed in many cities and used for different purposes such as Internet access, emergency and disaster recovery, security, health and medical systems, and public transportation $[1,2]$. At the same time, various research works dealing with WMNs have been conducted, and the IEEE 802.11s started the standardization of wireless LAN (WLAN) Mesh Networks after becoming a Task Group (TGs) in July 2004.

The HWMP protocol is proposed as the default path selection protocol within the IEEE 802.11 s draft [3, 4]. This protocol enables two different modes: on-demand path selection mode and proactive tree building mode. The proactive tree building mode constructs a routing tree topology by using either the PREQ (Path REQuest) mechanism or the proactive RANN (Route Announcement) mechanism when a root mesh node is configured.

Manuscript received February 9, 2013; revised June 9, 2013 and Dec. 20, 2013.

M. Guesmia and M. Guezouri are with the Signal Image Laboratory, Department of Electronics, Faculty of Electrical Engineering, University of Science and Technology, P.O. Box 1505, El-M'Naouer, Oran, ALGERIA (mohamed_guesmia,mguezouri)@yahoo.fr

N. Mbarek is with the Le2i (Laboratoire d'Electronique, Informatique et Image), Aile des Sciences de l'Ingénieur, 9, avenue Alain Savary, BP 47870, 21078 DIJON CEDEX, FRANCE (Nader.Mbarek@u-bourgogne.fr)
The resulting path within the routing tree topology is utilized by a mesh node to communicate with the root mesh node only. However, when a mesh node needs to communicate with any other mesh node (not playing the role of a root mesh node), the on-demand path discovery is initiated. The PREQ as well as the RANN transmission interval have an effect on the performance of the HWMP proactive tree building mode. Indeed, frequent messages transmission will cause high overhead but improves the routing table accuracy. On the other hand, high tree update interval may cause important delays for path recovery in case of a broken link.

In this paper we propose a new mechanism that enables to adjust dynamically the value of the PREQ transmission interval instead of a periodic fixed value as defined within the IEEE $802.11 \mathrm{~s}$ draft. The objective of our proposal is to provide WMN environment with up-to-date proactive trees while minimizing the overhead, in order to improve routing tables accuracy and path recovery delays. We compare our mechanism with the default value of PREQ transmission interval specified by the IEEE $802.11 \mathrm{~s}$ draft and implemented within the Network Simulator (NS-3) [5] for WMN according to a Waxman graph deployment of the backbone network based on IEEE 802.11s for multicast communication without QoS support. QoS multicast communication [6, 7, 8 and 9] issue could be easily implemented according to a minimum modification of our mechanism. To the best of our knowledge, no such study has been presented before to adapt dynamically the PREQ transmission interval.

The remainder of this paper is organized as follows. In section 2, we describe the IEEE 802.11s architecture for $\mathrm{WMN}(\mathrm{s})$. Section 3 presents our proposed mechanism and the corresponding simulation scenario using NS-3. This simulation aims to validate that our mechanism enables updating proactive trees with less overhead in order to improve mesh nodes routing table's accuracy and delays for path recovery. Section 4 concludes the paper and gives some future works.

\section{IEEE 802.11s ARCHITECTURE}

The IEEE 802.11 Task Group S (TGs) is standardizing wireless mesh networking based on IEEE 802.11 WLAN. The WMNs architecture is composed of three types of nodes [4, 5]: Mesh Point (MP), Mesh Access Point (MAP) and Mesh Portal Point (MPP), as illustrated in Figure 1. 


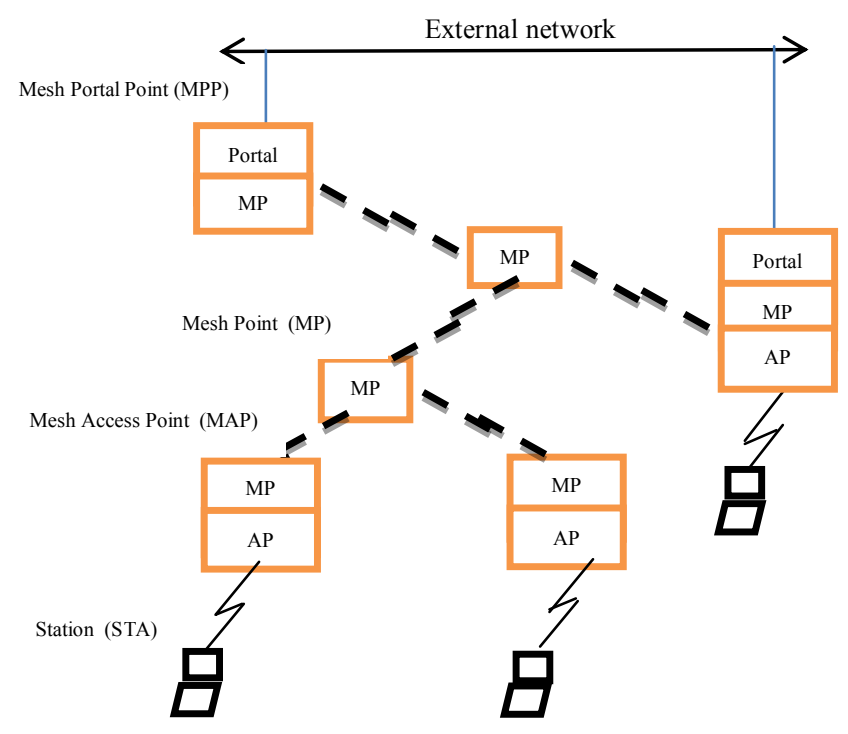

Figure 1. IEEE $802.11 \mathrm{~s}$ architecture

A Mesh Point (MP) supports wireless mesh services and participates in the interoperable operations within a mesh network, such as path selection and data forwarding. The Mesh Portal Point (MPP) acts as a gateway connecting the internal mesh network to the exterior, where Medium Access Control Service Data Units (MSDUs) enter/exit the mesh network from/to other networks. The MPP includes the MP functionality and could be co-located with an 802.11 portal and access point. The Mesh Access Point (MAP) is an MP playing the role of an access point: the MAP provides both mesh point and access point (AP) functionalities.

The backbone of the WMN(s) could support the internet access while dealing with multi-hop wireless communications. Thus, the WMN(s) benefit of all the advantages (mobility, wireless communications, easy deployment, etc.) and suffer of all the limitations (interferences, QoS requirements, etc.) of multi-hop wireless networking.

Moreover, the IEEE 802.11s draft [4] defines the HWMP protocol as the default path selection algorithm. The Air time Link Metric (ALM) is used by the HWMP protocol to select efficient paths. This metric is based on the transmission bit rate and error ratio. Air time link cost reflects the amount of channel resources consumed by transmitting the frame over a particular link. It is an approximate measure in order to make its implementation easier and to enhance interoperability [4]. The Air time link cost for every link is calculated as following:

$$
C_{a}=\left[o_{c a}+o_{p}+\frac{B_{t}}{r}\right] \frac{1}{1-e_{f}}
$$

Where $\mathrm{O}_{\text {ca }}, \mathrm{O}_{\mathrm{p}}$ and $\mathrm{B}_{\mathrm{t}}$ are constants, representing respectively the channel access overhead, the protocol overhead and the number of bits in a test frame. The input parameters $r$ and $e_{f}$ are respectively, the bit rate in Megabit per second $(\mathrm{Mb} / \mathrm{s})$ and the frame error ratio for the test frame with a size of $B_{t}$. The rate $r$ represents the rate at which the MP would transmit a frame of standard size $\left(B_{t}\right)$ based on current conditions. The frame error ratio $e_{f}$ is the probability that a frame of standard size $\left(B_{t}\right)$, transmitted at the current transmission bit rate $(\mathrm{r})$, is corrupted due to transmission error. The estimation of this ratio is a local implementation choice. routing:

The HWMP protocol offers two modes for information

- On demand path selection mode: it is an extension of the Ad-hoc On demand Distance Vector (AODV) protocol [10]. Within this selection mode, it is not necessary to have a node configured as root mesh station (STA). It uses peer to peer paths to establish communication between mesh stations.

- $\quad$ Proactive tree building mode: a node is configured as root mesh STA and a tree is build and maintained proactively. Mesh stations communicate via the tree paths. Maintaining the Integrity of the Specifications

\section{A. HWMP Mechanisms}

The IEEE 802.11s draft [4] defines, in the proactive tree building mode, two mechanisms for routing information:

- Proactive Path Request (PREQ): the root node (It could be an MP or a MAP [5]) periodically broadcasts a PREQ message. When an MP receives the PREQ message, it creates or updates the path towards the root node and it forwards the PREQ message as shown in Figure 2. The Path Reply (PREP) message is used to send path information to root node. This mode is well known as a two handshaking mechanism.
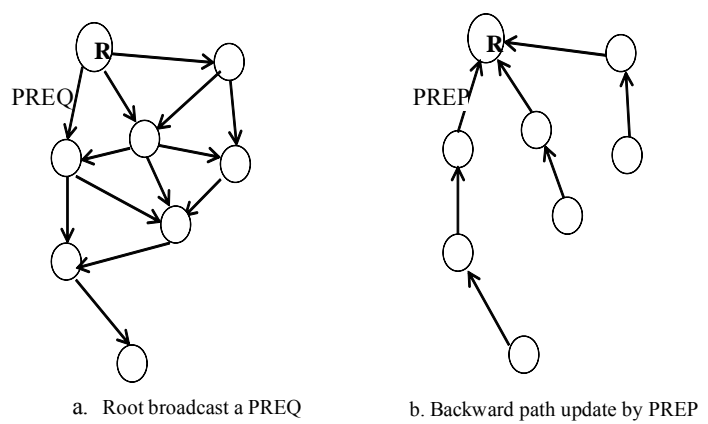

Figure 2. Proactive PREQ mechanism

- Proactive Root Announcement (RANN): the tree is build and maintained as illustrated by Figure 3 [11]. The root node periodically floods a RANN message in the network (see Figure 3a). Then an MP sends a 
unicast PREQ message to the root node in order to create or refresh a route (see Figure $3 b$ ). Finally, the root node replies using a PREP message towards the MP (see Figure 3c). This mode is well known as a three hand-shaking mechanism.

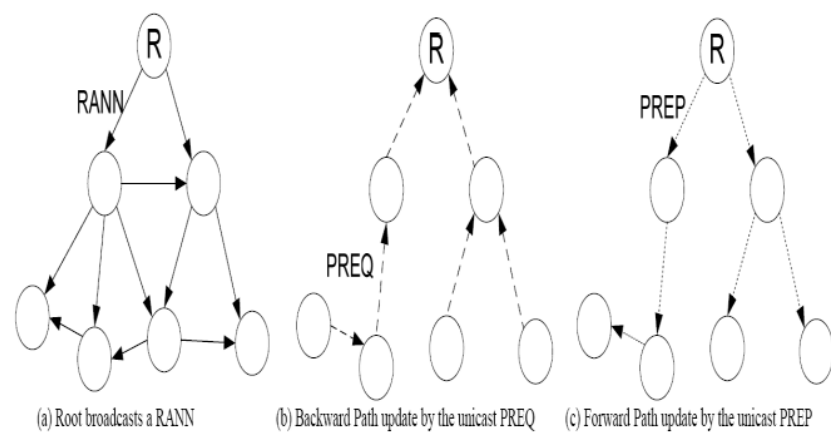

Figure 3. Proactive RANN mechanisms [11]

The interval time value of RANN or PREQ mechanisms transmission has an effect on the performance of the proactive tree building mode (construction of the tree routing topology). Indeed, with a short interval, the path reliability becomes high but the maintenance cost increases. On the other hand, if the interval time is long, the maintenance costs decreases (without guarantee of having an up-to-date path), but the communication delay between mesh points increases as the on-demand path discovery would be conducted when a link breakage occurs.

The root node sends proactive PREQ elements periodically. This period specifies the minimum interval of time (in TUs) during which a root node can send only one action frame, containing a proactive PREQ element. The default value given in the draft 802.11 s [4] is equal to 2000 TUs. According to $[12,13]$, only the proactive PREQ mechanism is used in $802.11 \mathrm{~s}$ implementation within the NS-3 network simulator.

\section{OUR PROPOSED MECHANISM}

A mesh network can be represented by a graph $G=$ $(\mathrm{V}, \mathrm{E})$ consisting of a set $\mathrm{V}$ of $\mathrm{N}=|\mathrm{V}|$ nodes, and a set $\mathrm{E}$ of $\mathrm{M}$ $=|\mathrm{E}|$ links [14]. Nodes can be a Mesh Point (MP), a Mesh Portal Point (MPP), a Mesh Access Point (MAP) or a client station (STA). Within the proposed mesh network environment, links represent the wireless communication links. Moreover, we only consider symmetric links and at most one link between a pair of nodes (single interface and single channel). A specific link in the set $\mathrm{E}$ between nodes $\mathrm{u}$ and $\mathrm{v}$ is denoted by $(\mathrm{u}, \mathrm{v})$. We denote by $G_{\text {HWMP }}=\left(V_{\text {HWMP }}\right.$, $E_{\text {HWMP }}$ ) the sub graph generated by the HWMP protocol. Thereby, $G_{\text {HWMP }}$ is a tree and don't contains cycles.

The basic idea of our proposed mechanism is to find an acceptable value for the PREQ transmission interval without changing the IEEE $802.11 \mathrm{~s}$ draft. Indeed, this interval, called dot11MeshHWMProotInterval within the IEEE 802.11s draft, is specified as a control variable with a Max-Access attribute equal to read-write. So, It could be written by an external management entity. Finding an acceptable value of this interval enables fewer transmissions of control messages and an up-to-date routing table within mesh nodes (less overhead and better transmission deadlines). We will determine this value will based on the weights of any eventual communications links of the entire WMN network as well as the links of the proactive tree generated by the HWMP protocol.

Every communication link between a pair of nodes $(u, v)$ has a weight. This weight represents the ALM metric value for this link. Therefore, the graph $G$ or the $G_{\text {HWMP }}$ tree has a weight which is the sum of all the links weights.

As the weight of a graph or a tree is an important characteristic [15], the weight ratio of the graph $G$ and the tree $G_{\text {HWMP }}$ will be used to assess the PREQ transmission interval value. The wireless environment has changing parameters and depends on several characteristics [16]. So, the value of the PREQ transmission interval will also be dynamic and based on the wireless environment characteristics, since the weight of $G$ and consequently the weight of $G_{\text {HWMP }}$ will vary over time.

Let $N_{1}$ and $N_{2}$ be the number of links (retrieved from the adjacent matrix) in $\mathrm{G}$ and $\mathrm{G}_{\mathrm{HWMP}}$ respectively. The value of $\mathrm{N}_{2}$ belongs to the interval [1, $\left.N_{1}-1\right]$. A value of $N_{2}$ equal to 1 means that there is at least a link between the root node and a mesh node (the proactive tree contains one link). The maximum value could not exceed $\mathrm{N}_{1}$ minus 1 to guarantee that $G_{\text {HWMP }}$ remains a tree.

We can then predict the value of the next PREQ transmission Interval (expressed in seconds) in a non-periodic manner according to, not only its default value in Time Units (TUs) specified within the IEEE 802.11s draft [4] and used by the network simulator NS-3 but also the size of both the graph $G$ representing all the WMN and the $G_{\text {HWMP }}$ proactive tree. Within the NS-3 implementation of the draft $802.11 \mathrm{~s}$, the PREQ transmission interval is equal to $1024 * 2000$, where 1024 is the Time Unit value used in the IEEE 802.11 standard and 2000 is the default value used by the root node to periodically broadcast PREQ elements as specified in the draft 802.11s [4].

In our proposed mechanism, we define a dynamic value for PREQ transmission interval according to formula (1):

$$
\text { PREQ transmission interval }=((T U) * \operatorname{int}(K)) / 100
$$

Where: TU is the Time Unit value, $\operatorname{int}(\mathrm{K}) \neq 0$ is the integer part of $\mathrm{K}$ and 


$$
\mathrm{K}=\frac{\text { (Average ALM of the graph } G \text { ) }}{\text { (Average ALM of the } G_{\text {HWMP }} \text { tree ) }}
$$

Where:

$$
\begin{aligned}
& \text { Average ALM of the graph } G=\sum_{e \in G} A L M(e) / N_{1} \\
& \text { Average ALM of the } G_{H W M P} \text { tree }=\sum_{\varepsilon \in G_{H W M P}} \operatorname{ALM}(e) / N_{2}
\end{aligned}
$$

Finally K can be expressed by:

$$
\mathrm{K}=\left(N_{2} / N_{1}\right) *\left(\sum_{e \in G} A L M(e) / \sum_{e \in G_{H W M P}} A L M(e)\right)
$$

The building of the HWMP proactive tree $G_{\text {HWMP }}$ depends on the connectivity of the WMN represented by the graph $G$. Therefore, the $N_{2}$ variable value in our mechanism depends on the $N_{1}$ variable value. Indeed, if we consider a fully connected graph $G$, then the maximum number of links can be computed by:

$$
N_{1}=\mathrm{N} *(\mathrm{~N}-1) / 2
$$

So the $G_{\text {HWMP }}$ proactive tree will be the spanning tree of $G$ with:

$$
N_{2}=N-1
$$

Then, our variable $\mathrm{K}$ used to determine the proposed dynamic transmission interval value will be computed by:

$$
K=(2 / N) *\left(\sum_{e \in G} A L M(e) / \sum_{e \in G_{H W M P}} A L M(e)\right)
$$

After considering the case of a fully connected graph, we describe in the following the case of a graph with only one link. In such mesh environment, the value on N1 is equal to 1 . Thus, the value of $\mathrm{N} 2$ will be equal to 1 . Using formula (2), $\mathrm{K}$ will be equal to 1 . In this particular situation and using formula (1) the update of the HWMP proactive tree (one link) will only depend on the fixed value of TU.

Figure 4 illustrates a simple example showing the benefits of specifying a dynamic value for the PREQ transmission interval instead of a fixed value as mentioned within the IEEE $802.11 \mathrm{~s}$ draft and implemented in the network simulator NS-3. Values on links for both the graph $G$ (Figure 4a) and the $G_{\text {HWMP }}$ proactive tree (Figure $4 \mathrm{~b}$ ) represent the ALM metric values. The node $\mathrm{R}$ represents the root node of the considered WMN topology.

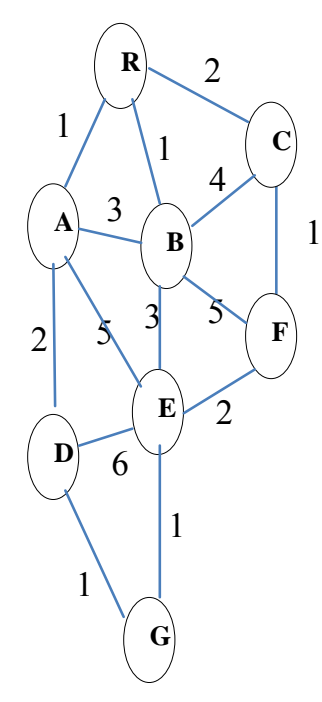

(a). Graph G mputing the PREQ d:

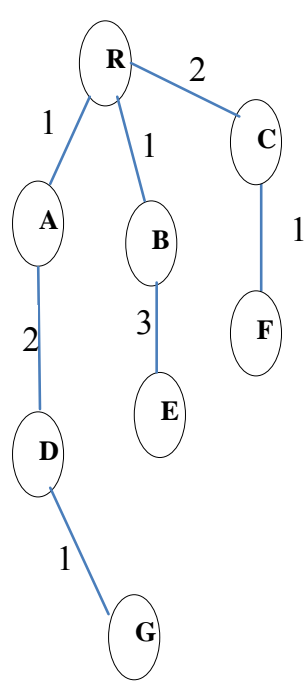

(b). $G_{\text {HWMP }}$ tree
Based on Figure 4, we can retrieve the weight of $G$ specified by: $\sum_{e \in G} A L M(e)=37$; but also the weight of $G_{H W M P}$ calculated as $\sum_{e \in G_{H W M P}} A L M(e)=11$. Moreover, using the formula (2), we obtain $\mathrm{K}=(7 / 14) *(37 / 11)=1.68$

For a simulation time duration equal, for example, to 240 seconds and using the transmission interval value adopted within the NS-3 network simulator $(2,048$ seconds $)$ in conformance with the default value specified in the IEEE 802.11 s draft, we obtain 117 updates of the HWMP proactive tree (240/2.048). Whereas, according to our proposed mechanism and using formula (1), we obtain a PREQ transmission interval equal to:

$$
\text { PREQ transmission interval }=((1024) * \operatorname{int}(1.68)) / 100
$$

Thereby, within the topology described in Figure 4 and using our mechanism, the PREQ transmission interval value is equal to 10.24 seconds. Our dynamic interval value will create 23 updates (240/10.24) of the HWMP proactive tree. Comparing the updates generated by our proposal to those generated by the default fixed value, it is clear that we have 94 $(117$ - 23) less updates of the HWMP proactive tree. Intuitively, we expect that our proposed mechanism will provide the WMN with less overhead and better end-to-end delays. The objective of the following simulation section is to validate and evaluate the performance of our dynamic PREQ interval proposal.

\section{A. Simulation environment:}

To evaluate our proposal, we performed simulations using Network Simulator NS-3 version 3.11 [5]. The simulation scenario was specified on a $500 \mathrm{~m} \times 500 \mathrm{~m}$ area in a singlechannel single interface environment with 30 mesh points which are deployed in a Waxman graph topology (nodes positions are established randomly). The Waxman graphs class 
belongs to the random graphs class, where the probability of link existence between two nodes decays exponentially with the geographic distance between those two nodes. Such graphs are often chosen because of their resemblance to actual network topologies. Each node has a transmission range of 100 $\mathrm{m}$ and the link bandwidth is set to $11 \mathrm{Mbps}$. We configure longdistance path for loss propagation model and we use a propagation delay model due to the interference within such environment. Table 1 gives an overview of the simulation environment.

Table 1. Simulation environment

\begin{tabular}{|l|l|}
\hline \multicolumn{1}{|c|}{ Field } & \multicolumn{1}{c|}{ Description } \\
\hline Area & $500 \mathrm{~m} * 500 \mathrm{~m}$ \\
\hline Total of nodes & 30 \\
\hline Kind of the topology deployment & Waxman graph \\
\hline Link speed & $11 \mathrm{Mbps}$ \\
\hline Root (multicast source node) & Mesh portal \\
\hline CBR transmission rate & $120 \mathrm{Kbps}$ \\
\hline Packet size & 1024 Bytes \\
\hline Network device & Single interface single channel \\
\hline Transmission range & $100 \mathrm{~m}$ \\
\hline
\end{tabular}

We consider for our simulation scenario, a multicast traffic generated within the wireless mesh network. For each multicast communication, the root node transmits 1024 bytes Multicast Constant Bit Rate (MCBR) packets to a set of destinations (chosen randomly) for a duration of 240 seconds and we run every simulation scenario 10 times. To be more realistic we inject background traffic within the wireless mesh network. The background traffic is generated as a UDP (User Datagram Protocol) traffic between a set nodes pairs chosen randomly. This background traffic starts 10 seconds before the beginning of the considered multicast traffic for a duration of 250 seconds.

As the HWMP protocol handles multicast communications as a unicast communications, the root node floods multicast data to nodes belonging to the proactive tree $G_{H W M P}$. Frequent updates of the $G_{\text {HWMP }}$ proactive tree guarantee the freshness of the considered tree at the expense of an important overhead within wireless mesh network. On the other hand, less updates will enhance the overhead without guarantee concerning the freshness of the $G_{\text {HWMP }}$ tree. We will compare our proposed mechanism (dynamic and non-periodic evaluation of the PREQ transmission interval) with the fixed value of NS-3 PREQ transmission interval (default NS-3 value is 2,048 seconds as defined in the IEEE $802.11 \mathrm{~s} \mathrm{draft}$ ).

According to our simulation scenario, a root node broadcasts multicast packets to all nodes within the mesh network. Indeed, it handles the multicast packets the same way it handles the broadcast packets. We evaluate our proposal by considering two metrics: Average total end-to-end delay and Control message overhead. For our simulation scenarios, these metrics are defined as follows:

- Average total end-to-end delay: the sum, for all communications, of received packets delays divided by the number of received packets.
- Control message overhead: the total number of control messages required to transfer multicast data.

\section{B. Simulation results:}

Based on 10 executions for every simulation scenario corresponding to the considered multicast group (chosen randomly and varying from 3 nodes members to 27 nodes members with a step of 3 nodes), with a duration of 240 seconds for every flow generated by the multicast group members, we obtain different results in terms of average total end-to-end delay and overhead.

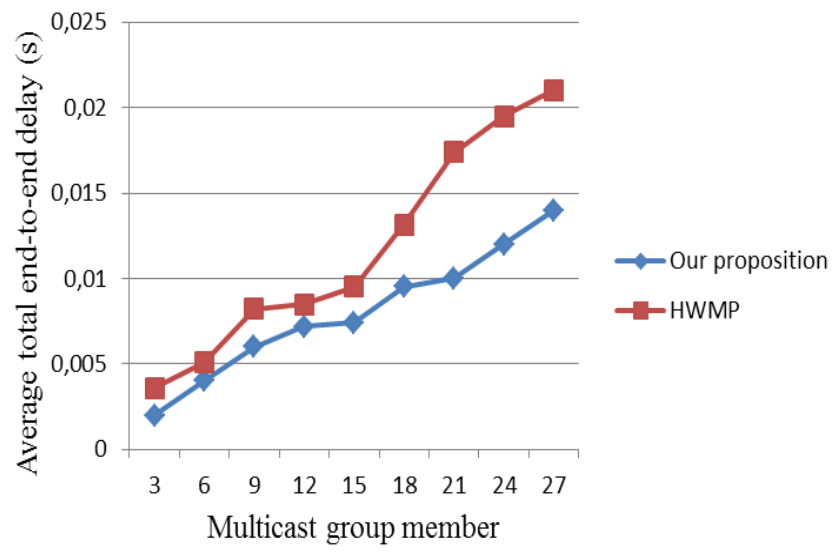

Figure 5. Average end-to-end delay

Figure 5 shows that using the fixed value of 2,048 seconds as a PREQ transmission interval (which is the default value within NS-3) leads to low performances, for all multicast flows, in terms of end-to-end delay compared to our dynamic and non-periodic PREQ transmission interval value. The overhead generated at a periodic interval (every 2,048 seconds) and with a fixed value doesn't take into account, neither nodes position and generated traffic, nor wireless environment changing characteristics. According to this fixed value, the $G_{\text {HWMP }}$ tree (the HWMP proactive tree) is built from the graph $G$ (the WMN network) every 2.048 seconds even if the $G_{\text {HWMP }}$ tree links are good and the routing tables are up-to-date. As shown in Figure 6, the overhead is significant only when the number of the multicast group members is important (greater than $70 \%$ of nodes belonging to the WMN network). Indeed, when the size of the multicast group is equal to 27 nodes which represents $90 \%$ of nodes that form the WMN network, our proposed dynamic interval, as shown in figure 5, enables an average total end-to-end delay less than 150 milliseconds which is an acceptable value for real time applications, whereas the classical HWMP protocol fails to provide real time applications with this QoS parameter guarantee (average total end-to-end is equal to 210 milliseconds) [17]. Moreover, when the size of the multicast group converge to the size of the nodes (30 nodes) belonging to the WMN network, the value of the weight of the $G_{\text {HWMP }}$ tree weight value is very close to the graph $G$ weight value. So, the PREQ transmission interval will converge to a 
fixed value implying that the $G_{H W M P}$ tree building will be done at almost regular interval throughout the simulation duration. According to Figure 6, the simulation scenarios with a multicast group number less than $90 \%$ of the entire WMN network size and using our proposed dynamic value enables low overhead. Indeed, our proposal takes into account the links characteristics based on the ALM metric while belonging to the WMN environment and the difference of weights between $G$ and $G_{H W M P}$ is still significant.

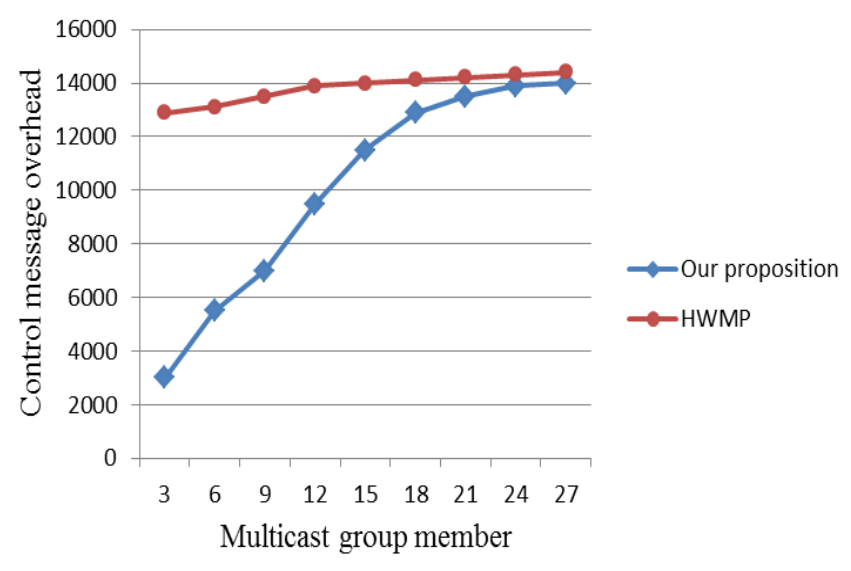

Figure 6. Control message overhead

The multicast scenarios considered in our simulations shows that our proposed mechanism based on a dynamic PREQ transmission interval depending on wireless mesh environment characteristics is scalable.

\section{C.Analysis of the computational complexity}

The calculation of the proactive tree through our dynamic mechanism will be at irregular intervals. Unlike the IEEE 802.11 s standard intervals which have a regular step, our mechanism depends on the wireless environment change.

At the initialization phase, the graph $\mathrm{G}$ is specified, and the $G_{\text {HWMP }}$ tree as well as the value of our PREQ transmission interval are computed according to formula (1).

Since it is a sum calculation, the complexity of specifying the $\mathrm{K}$ value according to formula (1) is $\mathrm{O}(\mathrm{N} 1 / \mathrm{N} 2)$, where $\mathrm{N} 1$ and $\mathrm{N} 2$ are defined in section 3. Considering formula (5), the corresponding complexity will be expressed in $\mathrm{O}(\mathrm{N})$. This linear complexity [18] is satisfying and does not require significant additional computation load compared to the fixed value of the PREQ transmission interval as defined in the IEEE 802.11 s standard.

\section{CONCLUSION AND FUTURE WORKS}

IEEE $802.11 \mathrm{~s}$ has been designed to support mesh configuration over IEEE 802.11 technology. The HWMP protocol has been adopted to provide routing in link layer. HWMP constructs a routing tree proactively which is maintained periodically. In this paper, we have enhanced the HWMP protocol by changing the PREQ transmission interval dynamically and in a non-periodic manner without any modification of the IEEE $802.11 \mathrm{~s}$ draft. Indeed, this interval was specified within the IEEE 802.11s draft as a control variable with a default value that could be changed by software designers or equipment constructors.

The objective of our proposed mechanism is to provide WMN with optimized fresh tree routing path (proactive trees) while minimizing total amount of overhead. The obtained simulation results show that a dynamic value for the PREQ transmission interval enhances the two performance parameters that we have evaluated, which are average total end-to-end delay and the control message overhead. According to our simulation scenarios, we tried to prove that a fixed value for PREQ transmission interval in such wireless environment and multicast traffic is not adapted for IEEE 802.11 s networks. On the other hand, we tried to provide critical applications (real time applications) with our proposed mechanism enabling better end-to-end delay and less overhead without any major modifications of the IEEE 802.11s draft. According to our simulation results, we can claim that our proposal is more adapted for QoS aware multicast traffic than the classical HWMP protocol. Moreover, the dynamic and non periodic value of the PREQ Transmission interval mechanism is scalable.

Due to the characteristics of wireless environment and as a perspective of this study, we will propose a framework to provide a model for the relationship between the graph categories representing the WMN backbone deployment and the PREQ transmission interval. As a second issue, we are working on a new simulation environment for QoS aware multicast routing subject to multiple constraints within 802.11 s based wireless mesh networks while taking into account different metrics with minimum modification concerning the variable $\mathrm{K}$ calculation in formula (1) in order to evaluate the dynamic PREQ transmission interval.

\section{REFERENCES}

[1] Lee, M., Zheng, J., KO, Y., Shrestha, D. (2006). "Emerging standards for wireless mesh technology", IEEE Wireless Communication.

[2] Akyildiz, L., Wang, X., Wang, W. (2005). "Wireless Mesh Networks: A Survey", Elsevier Computer Networks.

[3] Draft HWMP, doc.: IEEE 802.11-06/1778r1, http:// dev.laptop.org/ cscott/802.../11-06-1778-01-000s-hwmp-specification.doc, November 2006 .

[4] IEEE Standard for Information Technology--Telecommunications and information exchange between systems--Local and metropolitan area networks--Specific requirements Part 11: Wireless LAN Medium Access Control (MAC) and Physical Layer (PHY) specifications, Amendment 10: Mesh Networking, September 2011.

[5] Network Simulator 3, (September 2011). http://www.nsnam.org/.

[6] Khorov, E., Safonov, A. (2010). 'Multiple Metrics in Manet with End-ToEnd QoS Support for Unicast and Multicast Traffic', Proceedings of the International Workshop on Multiple Access Communications, Barcelona, Spain, 13-14 September, Springer, Vol. 6235, pp. 251-262.

[7] Bur, K., Ersoy, C. (2004). 'Multicast Routing for Ad Hoc Networks with a Multiclass Scheme for Quality of Service', Proceeding of the 19th International Symposium ISCIS 2004, Kemer-Antalya, Turkey, 27-29 October, Vol. 3280, pp. 187-197.

[8] Salama, H., Reeves, D., Viniotis, Y. (1997). 'Evaluation of Multicast Routing Algorithms for Real-Time Communication on High-Speed 
Networks', IEEE Journal on Selected Areas in Communications, Vol. 15 No.1, pp.332-345.

[9] Ben-Othman, J., Mokdad, L., Ould Cheikh, M. (2010) 'Q-HWMP: improving end to end QoS for 802.11s based mesh networks', Proceeding of the IEEE Globecom, Miami, Florida, USA, 6-10 December, E-ISBN : 978-14244-5637-6.

[10] Perkins, C., Belding-Royer, E., Das, S. (2003). "Ad hoc On-Demand Distance (AODV) Routing", RFC 3561.

[11] Bae, S., Ko, Y. (September 2010). "Efficient Layer-2 Multicasting for IEEE 802.11s based Wireless Mesh Networks", IEEE 978-1-4244-8086-9/10.

[12] Andreev, K. (August 2011). "Mesh module maintainer for NS-3", http://www.nsnam.org/developers/maintainer.

[13] Andreev, K., Boyko, P. (June 2011). "IEEE 802.11s Mesh Networking NS-3 Model", http://www.nsnam.org/workshops/wns3-2010/dot11s.pdf

[14] Kuipers, F.A. (2004). "Quality of service routing in the Internet: Theory, complexity and algorithms", $\mathrm{PhD}$ thesis, Delft University Press, The Netherlands.

[15] Spielman, D. (January 2012). Combinatorial Scientific Computing, Chapter 16, Spectral Graph Theory (Uwe Naumann, and Olaf Schenk) Chapman and Hall/CRC. ISBN:978-1-4398273-5-2

[16] Pahlavan, K., Krishnamurthy, P. (2001). Principles of Wireless Networks: A Unified Approach (First edition). Prentice Hall, Part of the Prentice Hall Communications Engineering and Emerging Technologies Series. ISBN-10: 0-13-093003-2.

[17] Chen, Y. Farley, T., Ye, N. (2004) 'QoS requirements of Network Applications on the Internet, Information Knowledge Systems Management 4, IOS Press, pp. 55-76.

[18] Sanjeev Arora and Boaz Barak, "Computational Complexity: A Modern Approach”, Princeton University, USA, January 2007.

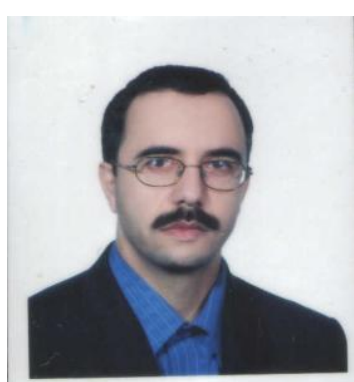

Mohamed GUESMIA is an assistant professor at University of Mostaganem, Mostaganem, Algeria since 2004. He received his Engineer degree (with honor) in Computer Science from University of Oran, Algeria in 1988, his M. Sc. Degree (with honor) in Computer Science from University of Sidi-Bel-Abbèss, Algeria in 2002. Currently he is a Ph.D. student in the Department of Computer Science at University of Oran. Research interests focus on study of the QoS multicast in the IEEE 802.11s based WMN. He is a member of the Signal Image Laboratory, Department of Electronics, Faculty of Electrical Engineering, University of Science and Technology, Oran, Algeria and a member of RAMoN project : Réseaux (Autonomie, Mobilité et Niveau de Service), Laboratoire d'Electronique, Informatique et Image (LE2I), University of Burgundy, Dijon, France.

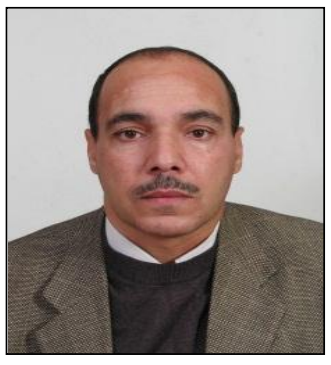

Mustapha GUEZOURI was born in Algeria in 1962. He received the engineer degree from the national Algerian institute of Telecommunications (ITOran) in 1987. He received the Master and $\mathrm{PhD}$ degrees in electrical engineering in 1990 and 2007, respectively from the Science and Technology University in Oran. Since 1990, he has been a Research Assistant at Signal and processing Laboratory, University of Oran. He is currently an Assistant Professor of electrical and computer engineering at the University of Oran, Algeria. His current research interests include Signal processing, neural network, mesh networks, VANET networks and computer security.

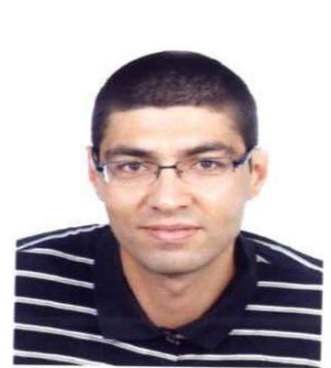

Nader MBAREK received his $\mathrm{PhD}$ degree in computer science from the University of Bordeaux, France in 2007, his M.S. in Networking from the University of Paris 6 (UPMC : University Pierre-et-Marie-Curie), France, in 2004 and his engineer degree in Telecommunications from the Higher School of Communication of Tunis (SUP'COM), Tunisia, in 2003. Currently, he is an associate professor at the ESIREM engineering school within the University of Burgundy. He leads the RAMoN project at the LE2I laboratory. This project concerns Service Level (QoS, Mobility and Security) guarantee, and Web Services technologies usage in self-management environment for IP networks. He is a reviewer for international journals and conferences and an expert for the French National Research Agency (ANR). 